\title{
Deuterated 3-Methylhistidine
}

National Cancer Institute

\section{Source}

National Cancer Institute. Deuterated 3-Methylhistidine. NCI Thesaurus. Code C91391.

A derivative of the amino acid histidine labeled with heavy hydrogen (D) used in diagnostic procedures. Upon intake of deuterated 3-methylhistidine (3-MH), this agent is incorporated into muscle protein and then is subsequently excreted unchanged in the urine. By measuring the amount of 3- $\mathrm{MH}$ in the urine, via analysis of deuterium, the rate of protein muscle catabolism can be determined and the risk of skeletal muscle atrophy or cachexia can be assessed. 3-methylhistidine is a myofibrillar-specific amino acid and is mainly found in muscle myosin and actin; proteolysis of myofibrils releases 3-MH that is excreted unchanged in the urine. 